\title{
Abelian state-closed subgroups of automorphisms of $m$-ary trees
}

\author{
Andrew M. Brunner and Said N. Sidki*
}

\begin{abstract}
The group $\mathcal{A}_{m}$ of automorphisms of a one-rooted $m$-ary tree admits a diagonal monomorphism which we denote by $x$. Let $A$ be an abelian state-closed (or self-similar) subgroup of $\mathcal{A}_{m}$. We prove that the combined diagonal and tree-topological closure $A^{*}$ of $A$ is additively a finitely presented $\mathbb{Z}_{m}[[x]]$-module, where $\mathbb{Z}_{m}$ is the ring of $m$-adic integers. Moreover, if $A^{*}$ is torsion-free then it is a finitely generated pro- $m$ group. Furthermore, the group $A$ splits over its torsion subgroup. We study in detail the case where $A^{*}$ is additively a cyclic $\mathbb{Z}_{m}[[x]]$-module, and we show that when $m$ is a prime number then $A^{*}$ is conjugate by a tree automorphism to one of two specific types of groups.
\end{abstract}

Mathematics Subject Classification (2010). 20E08, $20 \mathrm{~F} 18$.

Keywords. Automorphisms of trees, state-closed groups, self-similar groups, abelian groups, topological closure, $p$-adic integers, pro- $p$ groups.

\section{Introduction}

Automorphisms of one-rooted regular trees $\mathcal{T}(Y)$ indexed by finite sequences from a finite set $Y$ of size $m \geq 2$ have a natural interpretation as automata on the alphabet $Y$, with states which are again automorphisms of the tree. A subgroup of the group of automorphisms $\mathcal{A}(Y)$ of the tree is said to be state-closed in the language of automata (or self-similar in the language of dynamics) of degree $m$, provided that the states of its elements are themselves elements of the same group. If the group is not stateclosed then we may consider its state-closure. The prime example of a state-closed group is the group generated by the binary adding machine $\tau=(e, \tau) \sigma$, where $\sigma$ is the transposition $(0,1)$.

We study in this paper representations of general abelian groups as state-closed groups of degree $m$. For this purpose we use topological and diagonal closure oper-

\footnotetext{
${ }^{*}$ The first author is grateful for the hospitality of the Department of Mathematics of the University of Brasília as well as travel assistance from the University of Wisconsin-Parkside. The second author acknowledges support from the Brazilian scientific agencies CNPq and FAPDF. Both authors are thankful to Laurent Bartholdi for making numerous comments and the referee for a very careful reading of the paper.
} 
ations in the automorphism group of the tree. Representations of free abelian groups of finite rank as state-closed groups of degree 2 were characterized in [4].

An automorphism group $G$ of the tree group is said to be transitive, provided that the permutation group $P(G)$ induced by $G$ on the set $Y$ is transitive; actions of groups on sets will be applied on the right. It will be shown that the structure of state-closed groups can in a certain sense be reduced to those which are transitive.

The automorphism group $\mathcal{A}(Y)$ of the tree is a topological group with respect to the topology inherited from the tree. This topology allows us to exponentiate elements of $\mathcal{A}(Y)$ by $m$-ary integers from $\mathbb{Z}_{m}$. Given a subgroup $G$ of $\mathcal{A}(Y)$, its topological closure $\bar{G}$ with respect to the tree topology belongs to the same variety as $G$. Also, if $G$ is state-closed then so is $\bar{G}$.

The diagonal map $\alpha \rightarrow \alpha^{(1)}=(\alpha, \alpha, \ldots, \alpha)$ is a monomorphism of $\mathcal{A}_{m}$. Define inductively $\alpha^{(0)}=\alpha, \alpha^{(i+1)}=\left(\alpha^{(i)}\right)^{(1)}$ for $i \geq 0$. It is convenient to introduce a symbol $x$ and write $\alpha^{(i)}$ as $\alpha^{x^{i}}$ for $i \geq 0$. This will permit more general exponentiation, by formal power series $p(x) \in \mathbb{Z}_{m}[[x]]$. Given a subgroup $G$ of $\mathcal{A}(Y)$, its diagonal closure is the group $\widetilde{G}=\left\langle G^{(i)} \mid i \geq 0\right\rangle$. Observe that the diagonal closure operation preserves the state-closed property.

We will show that given an abelian transitive state-closed group $A$, its diagonal closure $\tilde{A}$ is again abelian. The composition of the diagonal and topological closures when applied to $A$ produces an abelian group denoted by $A^{*}$, which can be viewed additively as a finitely generated $\mathbb{Z}_{m}[[x]]$-module. This approach was first used in [2].

The prime decomposition $m=\prod_{1 \leq i \leq s} p_{i}^{k_{i}}$ provides us with the decomposition $\mathbb{Z}_{m}=\bigoplus_{1 \leq i \leq s} \varepsilon_{i} \mathbb{Z}_{p_{i}} k_{i}$, where $\varepsilon_{i}$ are orthogonal idempotents such that $1=$ $\sum_{1 \leq i \leq s} \varepsilon_{i}$, and also gives us the decomposition $\mathbb{Z}_{m}[[x]]=\bigoplus_{1 \leq i \leq s} \varepsilon_{i} \mathbb{Z}_{p_{i}} k_{i}[[x]]$. When $m=p^{k}$ and $p$ a prime number, the rings $\mathbb{Z}_{m}[[x]]$ and $\mathbb{Z}_{p}[[x]]$ are isomorphic, yet when $k>1$ they are different representations of the same object and for this reason we distinguish between them.

In Sections 3 and 4 we prove

Theorem 1. Let $A$ be an abelian transitive state-closed group of degree $m$. Then

(1) the group $A^{*}$ is isomorphic to a finitely presented $\mathbb{Z}_{m}[[x]]$-module;

(2) if $A^{*}$ is torsion-free then it is a finitely generated $\mathbb{Z}_{m}$-module which is also a pro-m group.

Item (1) is part of Theorem 5 and item (2) is Corollary 1 of Theorem 6.

We consider in Section 5 torsion subgroups of state-closed abelian groups and use methods from virtual endomorphisms of groups (see [3], [1]; reviewed in Section 5.1) to prove the following structural result.

Theorem 2. Let $A$ be an abelian transitive state-closed group of degree $m$ and $\operatorname{tor}(A)$ its torsion subgroup. Then 
(i) $\operatorname{tor}(A)$ is a direct summand of $A$ and has exponent a divisor of the exponent of $P(A)$;

(ii) the action of $A$ on the $m$-ary tree induces transitive state-closed representations of $\operatorname{tor}(A)$ on the $m_{1}$-tree and of $\frac{A}{\operatorname{tor}(A)}$ on the $m_{2}$-tree, where $m_{1}=|P(\operatorname{tor}(A))|$ and $m_{2}=\left|\frac{P(A)}{P(\operatorname{tor}(A))}\right|$

(iii) if $A=\operatorname{tor}(A)$ and $P(A) \cong \bigoplus_{1 \leq i \leq k} \frac{\mathbb{Z}}{m_{i} \mathbb{Z}}$, then $A^{*} \cong \bigoplus_{1 \leq i \leq k} \frac{\mathbb{Z}}{m_{i} \mathbb{Z}}[[x]]$.

The above results are analogous to Theorem 4.3.4 of [5] on the structure of finitely generated pro- $p$ groups. By item (i) of the theorem, an abelian torsion group $G$ of infinite exponent cannot have a faithful representation as a transitive state-closed group for any finite degree. Put differently, the group $G$ does not admit any simple virtual endomorphism. On the other hand, the group of automorphisms of the $p$-adic tree is replete with abelian $p$-subgroups of infinite exponent. Item (iii) follows from Theorem 7, which is a conjugacy result and therefore more general than isomorphism.

We focus our attention in Section 6 on transitive state-closed abelian groups $A$, for which $A^{*}$ is additively a cyclic $\mathbb{Z}_{m}[[x]]$-module. We show

Theorem 3. (1) Let $q_{1}, \ldots, q_{\boldsymbol{m}} \in \mathbb{Z}_{m}[[x]]$ and let $\sigma$ be the cycle $(1,2, \ldots, m)$. Then the expression

$$
\alpha=\left(\alpha^{q_{1}}, \ldots, \alpha^{q_{m}}\right) \sigma
$$

is a well-defined automorphism of the m-ary tree and the state-closure $A$ of $\langle\alpha\rangle$ is an abelian transitive group. The group $A^{*}$ is additively isomorphic to the quotient ring $\frac{\mathbb{Z}_{m}[[x]]}{(r)}$, where

$$
r=m-x q \text { and } q=q_{\mathbf{1}}+\cdots+q_{\boldsymbol{m}} .
$$

(2) Let $A$ be a transitive state-closed abelian group of degree $m$ such that $A^{*}$ is additively a cyclic $\mathbb{Z}_{m}[[x]]-$ module. Then $P(A)$ is cyclic, say generated by $\sigma$, and $A^{*}$ is the state-diagonal-topological closure of an element of the form $\alpha=\left(\alpha^{q_{1}}, \ldots, \alpha^{q_{m}}\right) \sigma$ for some $q_{1}, \ldots, q_{\boldsymbol{m}} \in \mathbb{Z}_{m}[[x]]$.

Finally we provide a complete description of the group $A^{*}$ for state-closed groups of prime degree. Let $j \geq 1$ and let $D_{m}(j)$ be the group generated by the set of states of the generalized adding machine $\alpha=\left(e, \ldots, e, \alpha^{x^{j-1}}\right) \sigma$ acting on the $m$-ary tree with $\sigma=(1,2, \ldots, m)$. The topological closure of $D_{m}(j)$ seen as $\mathbb{Z}_{m}$-module is isomorphic to the ring $\frac{\mathbb{Z}_{m}[[x]]}{(r)}, r=m-x^{j}$.

Theorem 4. Let $A$ be an abelian transitive state-closed group of prime degree $m$ and let $\sigma$ be the $m$-cycle automorphism. If $\operatorname{tor}(A)$ is nontrivial then $A^{*}$ is a torsion group conjugate to $\langle\sigma\rangle^{*}\left(\cong \frac{\mathbb{Z}}{m \mathbb{Z}}[[x]]\right)$. If $A$ is torsion-free then $A^{*}$ is a torsion-free group conjugate to the topological closure of $D_{m}(j)$ for some $j$.

One of the questions that has remained unanswered is whether a free abelian group of infinite rank admits a faithful transitive state-closed representation, even of prime degree. 


\section{Preliminaries}

We fix the notation $Y=\{1,2, \ldots, m\}, \mathcal{T}_{m}=\mathcal{T}(Y), \mathcal{A}_{m}=\mathcal{A}(Y)$ and we let $\operatorname{Perm}(Y)$ be the group of permutations of $Y$. A permutation $\gamma \in \operatorname{Perm}(Y)$ is extended to an automorphism of the tree by $\gamma: y u \rightarrow y^{\gamma} u$, fixing the non-initial letters of every sequence. An automorphism $\alpha \in \mathcal{A}_{m}$ is represented as $\alpha=\left(\alpha_{1}, \alpha_{2}, \ldots, \alpha_{m}\right) \sigma(\alpha)$, where $\alpha_{i} \in \mathcal{A}_{m}$ and $\sigma(\alpha) \in \operatorname{Perm}(Y)$. Successive developments of $\alpha_{i}$ produce for us $\alpha_{u}$ (a state of $\alpha$ ) for every finite string $u$ over $Y$. is

The product of $\alpha=\left(\alpha_{1}, \alpha_{2}, \ldots, \alpha_{m}\right) \sigma(\alpha)$ and $\beta=\left(\beta_{1}, \beta_{2}, \ldots, \beta_{m}\right) \sigma(\beta)$ in $\mathcal{A}_{m}$

$$
\alpha \beta=\left(\alpha_{1} \beta_{(1) \sigma(\alpha)}, \ldots, \alpha_{m} \beta_{(m) \sigma(\alpha)}\right) \sigma(\alpha) \sigma(\beta) .
$$

Let $G$ be a subgroup of $\mathcal{A}_{m}$. Denote the subgroup of $G$ which fixes the vertices of the $i$-th level of the tree by $\operatorname{Stab}_{G}(i)$. Given $y \in Y$, denote by $\operatorname{Fix}_{G}(y)$ the subgroup of $G$ consisting of the elements of $G$, which fix $y$. The group $G$ is said to be recurrent provided it is transitive and $\operatorname{Fix}_{G}(1)$ projects in the 1st coordinate onto $G$.

The group $\mathcal{A}_{m}$ is the inverse limit of its quotients by the $i$-th level stabilizers $\operatorname{Stab}_{\mathcal{A}_{m}}(i)$ of the tree and is as such a topological group where each $\operatorname{Stab}_{\mathcal{A}_{m}}(i)$ is an open and closed subgroup. For a subgroup $G$ of automorphisms of the tree, its topological closure $\bar{G}$ coincides with the set of all infinite products $\ldots g_{i} \ldots g_{1} g_{0}$, or alternately, $g_{0} g_{1} \ldots g_{i} \ldots$, where $g_{i} \in \operatorname{Stab}_{G}(i)$. The group $\bar{G}$ satisfies the same group identities as $G$. We note that the property of being state-closed is also preserved by the topological closure operation.

Let $\alpha$ be an automorphism of the tree. Then $\langle\bar{\alpha}\rangle=\left\{\alpha^{p} \mid p \in \mathbb{Z}_{m}\right\}$. More generally, for $q=\sum_{i \geq 0} q_{i} x^{i} \in \mathbb{Z}_{m}[[x]]$ with $q_{i} \in \mathbb{Z}_{m}$, we write the expression

$$
\alpha^{q}=\alpha^{q_{0}} \alpha^{q_{1} x} \ldots \alpha^{q_{i} x^{i}} \ldots,
$$

which can be verified to be a well-defined automorphism of the tree.

We recall the reduction of group actions to transitive ones, with a view to a similar reduction for state-closed groups of automorphisms of trees. Let $G$ be a subgroup of Perm $(Y)$, let $\left\{Y_{i} \mid i=1, \ldots, s\right\}$ be the set of orbits of $G$ on $Y$ and let $\left\{\rho_{i}: G \rightarrow \operatorname{Perm}\left(Y_{i}\right) \mid i=1, \ldots, s\right\}$ be the set of induced representations. Then, each $\rho_{i}$ is transitive and $\rho: G \rightarrow \prod_{1 \leq i \leq s} \operatorname{Perm}\left(Y_{i}\right) \leq \operatorname{Perm}(Y)$ defined by $g \rightarrow\left(g^{\rho_{1}}, \ldots, g^{\rho_{s}}\right)$ is a monomorphism. The reduction for tree actions follows from

Lemma 1. Let $G$ be a state-closed group of automorphisms of the tree $\mathcal{T}(Y)$ and let $X$ be a $P(G)$-invariant subset of $Y$. Then $\mathcal{T}(X)$ is $G$-invariant and for the resulting representation $\mu: G \rightarrow \mathcal{A}(X)$ the group $G^{\mu}$ is state-closed. If $G$ is diagonally closed or is topologically closed then so is $G^{\mu}$.

Proof. Let $x u$ be a sequence from $X$ and let $\alpha \in G$. Then $(x u)^{\alpha}=x^{\sigma(\alpha)} u^{\alpha_{x}}$. As $x^{\sigma(\alpha)} \in X$ and $\alpha_{x} \in G$, it follows that $(x u)^{\alpha}$ is a sequence from $X$. Also, for any sequence $u$ from $X$, we have $\left(\alpha^{\mu}\right)_{u}=\left(\alpha_{u}\right)^{\mu}$. Thus, $G^{\mu}$ is state-closed. The last assertion is clear. 
We note the following important properties of transitive state-closed abelian groups $A$.

Proposition 1. Let $A$ be an abelian transitive state-closed group of degree $m$. Then $\operatorname{Stab}_{A}(i) \leq A^{(i)}$ for all $i \geq 0$. The group $\tilde{A}$ is an abelian transitive state-closed group and is a minimal recurrent group containing $A$. Moreover, the topological closure and diagonal closure operations commute when applied to A. The diagonal-topological closure $A^{*}$ of $A$ is an abelian transitive state-closed group.

Proof. Let $\alpha=\left(\alpha_{1}, \ldots, \alpha_{m}\right) \sigma, \beta=\left(\beta_{1}, \ldots, \beta_{m}\right) \in A$. Then the conjugate of $\beta$ by $\alpha$ is

$$
\beta^{\alpha}=\left(\beta_{1}^{\alpha_{1}}, \ldots, \beta_{m}^{\alpha_{m}}\right)^{\sigma}
$$

As $\alpha_{i}, \beta_{i} \in A$ and $A$ is abelian, it follows that $\beta=\left(\beta_{1}, \ldots, \beta_{m}\right)^{\sigma}$. Furthermore, since $A$ is transitive, $\beta=\left(\beta_{1}, \ldots, \beta_{1}\right)=\left(\beta_{1}\right)^{(1)}$. Thus, $\operatorname{Stab}_{A}(i) \leq A^{(i)}$ for all $i$. A similar verification shows that $\tilde{A}=\left\langle A^{(i)} \mid i \geq 0\right\rangle$ is abelian.

Let $G$ be a recurrent group such that $A \leq G \leq \tilde{A}$. Given $\alpha \in G$, as $G$ is recurrent, there exists $\beta \in \operatorname{Stab}_{G}(1)$ such that $\beta=\left(\beta_{1}, \ldots, \beta_{m}\right)$ with $\beta_{1}=\alpha$. Since $G$ is transitive and abelian, we have $\beta_{1}=\cdots=\beta_{m}=\alpha$; that is, $\beta=\alpha^{(1)}$. Hence, $A^{(i)} \leq G$ and $G=\tilde{A}$ follows.

The last two assertions of the proposition are clear.

The following result indicates the smallness of recurrent transitive abelian groups from the point of view of centralizers.

Proposition 2 (Theorem 7 [1]). (1) Let $A$ be a recurrent abelian group of degree $m$ and let $C_{\mathcal{A}_{m}}(A)$ be the centralizer of $A$ in $\mathcal{A}_{m}$. Then $C_{\mathcal{A}_{m}}(A)=\bar{A}$.

(2) Let $m$ be a prime number and $A$ be an infinite transitive state-closed abelian group. Then $C_{\mathcal{A}_{m}}(A)=\bar{A}$.

This result will be used in the proofs of Lemma 3 and step 4 of Theorem 9.

\section{A presentation for $A^{*}$}

Let $A$ be a transitive abelian state-closed group of degree $m$ and let $A^{*}$ be its diagonaltopological closure. Then $A^{*}$ is additively a $\mathbb{Z}_{m}[[x]]$-module having the following properties. Given $\alpha \in A^{*}$, then

(i) $x \alpha=0$ implies $\alpha=0$;

(ii) $m \alpha=x \gamma$ for some $\gamma \in A^{*}$.

Let $P(A)$ be given by its presentation

$$
\left.\left\langle\sigma_{i}(1 \leq i \leq k)\right| \sigma_{i}^{m_{i}}=e, \text { abelian }\right\rangle .
$$


Choose for each $\sigma_{i}$ an element $\beta_{i}$ in $A$, which induces $\sigma_{i}$ on $Y$; denote $\beta_{i}$ by $\beta\left(\sigma_{i}\right)$. Then, for any $n \geq 0$, the automorphism of the tree $\beta\left(\sigma_{i}\right)^{(n)}$ is an element of $\tilde{A}$ which induces $\left(\sigma_{i}\right)^{(n)}$ on the $(n+1)$-th level of the tree. Although the notation $\beta_{i}$ has been used to indicate the $i$ th entry in an automorphism $\beta$, we hope this new usage will not cause confusion.

Theorem 5. Let $A$ be a transitive abelian state-closed group of degree $m$. Then $A^{*}$ is additively a $\mathbb{Z}_{m}[[x]]$-module generated by

$$
\left\{\beta_{i} \mid 1 \leq i \leq k\right\}
$$

subject to the set of defining relations

$$
\left\{r_{i}=\sum_{1 \leq j \leq k} m_{i} \beta_{i}-p_{i j} \beta_{j} x=0 \mid 1 \leq i \leq k\right\} \text { for some } p_{i j} \in \mathbb{Z}_{m}[[x]] .
$$

Moreover, there exist $r, q \in \mathbb{Z}_{m}[[x]]$ such that $r=m-x q$ and $r A^{*}=(0)$. The elements of $A^{*}$ can be represented additively as $\sum_{1 \leq i \leq k} p_{i} \beta_{i}$, where $p_{i}=\sum_{j \geq 0} p_{i j} x^{j}$ and each $p_{i j} \in \mathbb{Z}$ with $0 \leq p_{i j}<m$.

Proof. Let $\alpha \in A^{*}$ and $\sigma(\alpha)=\prod_{1 \leq i \leq k} \sigma_{i}^{r_{i 1}}, 0 \leq r_{i 1}<m_{i}$. Then either $\alpha\left(\prod_{1 \leq i \leq k} \beta_{i}^{r_{i 1}}\right)^{-1}$ is the identity element or there exists $l_{2} \geq 1$ such that

$$
\alpha\left(\prod_{1 \leq i \leq k} \beta_{i}^{r_{i 1}}\right)^{-1} \in \operatorname{Stab}\left(l_{2}\right) \backslash \operatorname{Stab}\left(l_{2}+1\right)
$$

and so, $\alpha\left(\prod_{1 \leq i \leq k} \beta_{i}^{r_{i 1}}\right)^{-1}=(\gamma)^{\left(l_{2}\right)}$ for some $\gamma \in A^{*}$. We treat $\gamma$ in the same manner as $\alpha$. In the limit, we obtain

$$
\alpha=\prod_{1 \leq i \leq k}\left(\beta_{i}^{r_{i 1}}\left(\beta_{i}^{r_{i 2}}\right)^{\left(l_{2}\right)} \ldots\left(\beta_{i}^{r_{i j}}\right)^{\left(l_{j}\right)} \ldots\right)=\prod_{1 \leq i \leq k} \beta_{i}^{q_{i}},
$$

where $0 \leq r_{i j}<m_{i}, 1 \leq l_{2}<l_{3}<\cdots<l_{j}<\cdots$, and $q_{i}=r_{i 1}+\sum_{j \geq 2} r_{i j} x^{l_{j}}$ are formal power series in $x$. Additively we then have

$$
\alpha=\sum_{1 \leq i \leq k} q_{i} \beta_{i} \in \sum_{1 \leq i \leq k} \mathbb{Z}_{m_{i}}[[x]] \beta_{i} .
$$

Each relation $\sigma_{i}^{m_{i}}=e$ in $P$ produces in $A^{*}$ a relation of the form

$$
\beta_{i}^{m_{i}}=\prod_{1 \leq j \leq k} \beta_{j}^{x p_{i j}},
$$

where $p_{i j}$ are elements in the power series, as above; when written additively $\beta_{i}^{m_{i}}$ has the form

$$
m_{i} \beta_{i}=x\left(\sum_{1 \leq j \leq k} p_{i j} \beta_{j}\right) .
$$


Let $F=\bigoplus_{1 \leq i \leq k} \mathbb{Z}_{m}[[x]] \dot{\beta}_{i}$ be a free $\mathbb{Z}_{m}[[x]]$-module of rank $k$. Define the $\mathbb{Z}_{m}[[x]]-$ homomorphism

$$
\phi: \sum_{1 \leq i \leq k} \mathbb{Z}_{m}[[x]] \dot{\beta}_{i} \rightarrow A^{*}, \quad \sum_{1 \leq i \leq k} p_{i} \dot{\beta}_{i} \mapsto \prod_{1 \leq i \leq k} \beta_{i}^{p_{i}},
$$

and let $R$ be the kernel $\phi$. Define $J$ to be the $\mathbb{Z}_{m}[[x]]$-submodule of $R$ generated by

$$
\dot{r}_{i}=m_{i} \dot{\beta}_{i}-x\left(\sum_{1 \leq j \leq k} p_{i j} \dot{\beta}_{j}\right) \quad(1 \leq i \leq k) .
$$

We will show that $J=R$. So let $v \in R$ and write $v=\sum_{1 \leq i \leq k} \nu_{i} \dot{\beta}_{i}$, where

$$
v_{i}=\sum_{j \geq 0} v_{i j} x^{j}, \quad v_{i j}=v_{i j, 0}+m w_{i j} \in \mathbb{Z}_{m}
$$

Then $m_{i} \mid v_{i 0,0}, v_{i 0,0}=m_{i} v_{i 0,0}^{\prime}$; factor $m=m_{i} m_{i}^{\prime}$. Therefore,

$$
\begin{aligned}
v_{i} & =v_{i 0}+\left(\sum_{j \geq 1} v_{i j} x^{j-1}\right) x, \\
v_{i 0} & =m_{i} v_{i 0,0}^{\prime}+m w_{i 0}=\left(v_{i 0,0}^{\prime}+m_{i}^{\prime} w_{i 0}\right) m_{i}, \\
v_{i} \dot{\beta}_{i} & =\left(v_{i 0,0}^{\prime}+m_{i}^{\prime} w_{i 0}\right)\left(m_{i} \dot{\beta}_{i}\right)+\left(\sum_{j \geq 1} v_{i j} x^{j-1}\right) x \dot{\beta}_{i}, \\
& \equiv\left(v_{i 0,0}^{\prime}+m_{i}^{\prime} w_{i 0}\right)\left(x \sum_{1 \leq j \leq k} p_{i j} \dot{\beta}_{j}\right)+\left(\sum_{j \geq 1} v_{i j} x^{j-1}\right) x \dot{\beta}_{i} \bmod J .
\end{aligned}
$$

Hence

$$
v=\sum_{1 \leq i \leq k} v_{i} \dot{\beta}_{i} \in x \mu+J, \quad \mu=\sum_{1 \leq i \leq k} \mu_{i} \dot{\beta}_{i} \in R .
$$

Hence, by repeating the argument, we obtain

$$
v \in\left(\bigcap_{i \geq 1} x^{i} R\right)+J=J, \quad J=R .
$$

On re-writing the relations $m_{i} \beta_{i}=\sum_{1 \leq j \leq k} p_{i j} x \beta_{j}$ in the form

$$
p_{i 1} x \beta_{1}+\cdots+\left(p_{i i} x-m_{i}\right) \beta_{i}+\cdots+p_{k k} x \beta_{k}=0
$$

we see that the $k \times k$ matrix of coefficients of these equations has determinant $r=$ $m-x q$ for some $q \in \mathbb{Z}_{m}[[x]]$ and thus $r$ annuls $A^{*}$.

The last assertion of the theorem follows by using $r=m-q x \in R$ to reduce the coefficients modulo $m$.

\section{The $m$-congruence property}

A group $G$ of automorphisms of the $m$-ary tree is said to satisfy the $m$-congruence property, provided that given $m^{i}$ there exists $l(i) \geq 1$ such that $\operatorname{Stab}_{G}(l(i)) \leq G^{m^{i}}$ for all $i$, in which case the topology on $G$ inherited from $\mathcal{A}(Y)$ is equal to the pro- $m$ topology. Since when $A^{*}$ is written additively, we have $\operatorname{Stab}_{G}(l(i))=x^{l(i)} A^{*}$, the $m$-congruence property reads $x^{l(i)} A^{*} \leq m^{i} A^{*}$. 
Theorem 6. Let $r=m-q x^{j} \in \mathbb{Z}_{m}[[x]]$ with $q \in \mathbb{Z}_{m}[[x]]$ and $j \geq 1$. Let $S$ be quotient ring $\frac{\mathbb{Z}_{m}[[x]]}{(r)}$. Suppose that $S$ is torsion-free. Then $S$ is a finitely generated pro-m group.

Proof. From the decomposition $\mathbb{Z}_{m}[[x]]=\bigoplus_{1 \leq i \leq s} \varepsilon_{i} \mathbb{Z}_{p_{i} k_{i}}[[x]]$ corresponding to the prime decomposition $m=\prod_{1 \leq i \leq s} p_{i}^{k_{i}}$, we obtain

$$
\begin{aligned}
r & =\sum_{1 \leq i \leq s} r_{i}, \\
r_{i} & =\varepsilon_{i} r=p_{i}^{k_{i}}-q_{i}(x) x^{j}, \\
S & =\sum_{1 \leq i \leq s} S_{i}, \quad S_{i}=\frac{\mathbb{Z}_{p_{i}^{k_{i}}}^{[[x]]}}{\left(r_{i}\right)},
\end{aligned}
$$

where each $S_{i}$ is torsion-free. Thus, it is sufficient to address the case where $m$ is a prime power $p^{k}$.

(1) First, we show that $S$ is a pro- $m$ group.

So let $r=p^{k}-q x^{j}$ and decompose $q=q(x)=s(x)+p \cdot t(x)$, where each non-zero coefficient of $s(x)$ is an integer relatively prime to $p$. If $s(x)=0$ then $q(x)=p \cdot t(x)$ and

$$
r=p^{k}-q(x) x^{j}=p^{k}-p \cdot t(x) x^{j}=p\left(p^{k-1}-t(x) x^{j}\right) \in(r) ;
$$

but as by hypothesis $S$ is torsion free, we have $p^{k-1}-t(x) x^{j} \in(r)$, which is not possible.

Write $s(x)=x^{l} u(x)$, where $l \geq 0$ and $u(x)$ is invertible in $\mathbb{Z}_{m}[[x]]$ with inverse $u^{\prime}(x)$. Then $q(x)=x^{l} u(x)+p \cdot t(x)$ and

$$
r=p^{k}-\left(x^{l} u(x) x^{j}+p \cdot t(x) x^{j}\right)=p\left(p^{k-1}-t(x) x^{j}\right)-x^{j+l} u(x) .
$$

Therefore, on multiplying by $u^{\prime}(x)$, the inverse of $u(x)$, we obtain

$$
p\left(p^{k-1}-t(x) x^{j}\right) u^{\prime}(x) \equiv x^{j+l} \bmod r .
$$

It follows that

$$
x^{j+l} S \leq p S, \quad x^{n(j+l)} S \leq p^{n} S .
$$

(2) Now we show that $S$ is finitely generated as a $\mathbb{Z}_{m}$-module.

By the previous step there exist $l \geq 1$ and $v(x) \in \mathbb{Z}[[x]]$ such that

$$
x^{l} \equiv m v(x) \bmod r .
$$

Decompose $v(x)=v_{1}(x)+v_{2}(x) x^{l}$ where the degree of $v_{1}(x)$ is less that $l$. Then 
we deduce modulo $r$ :

$$
\begin{aligned}
v(x) & \equiv v_{1}(x)+v_{2}(x) m v(x), \\
v_{2}(x) v(x) & \equiv w(x) \in \mathbb{Z}[[x]], \\
w(x) & =w_{1}(x)+w_{2}(x) x^{l}, \\
v(x) & \equiv v_{1}(x)+m w(x) \\
& \equiv v_{1}(x)+m w_{1}(x)+m w_{2}(x) x^{l} \\
& \vdots \\
v(x) & \equiv a_{0}+a_{1} x+\cdots+a_{(l-1)} x^{l-1}, \quad a_{i} \in \mathbb{Z}_{m} .
\end{aligned}
$$

We have shown that $S$ is generated by $1, x, \ldots, x^{l-1}$ as a pro- $m$ group.

Corollary 1. Let $A$ be an abelian transitive state-closed group of degree $m$. Suppose that the group $A^{*}$ is torsion-free. Then $A^{*}$ is a finitely generated pro-m group.

Proof. With previous notation, the group $A^{*}$ is a $\mathbb{Z}_{m}[[x]]$-module generated by

$$
\left\{\beta_{i}=\beta\left(\sigma_{i}\right) \mid 1 \leq i \leq k\right\}
$$

and is annihilated by $r=m-q x^{j} \in \mathbb{Z}_{m}[[x]]$ for some $q \in \mathbb{Z}_{m}[[x]]$ and $j \geq 1$.

It follows that $A^{*}$ is an $S$-module, where $S=\frac{\mathbb{Z}_{m}[[x]]}{(r)}$. Since $S$ satisfies the $m$-congruence property, it follows that $A^{*}$ is a pro- $m$ group.

That $A^{*}$ is a finitely generated $\mathbb{Z}_{m}$-module, is a consequence of $S$ being a finitely generated $\mathbb{Z}_{m}$-module.

\section{Torsion in state-closed abelian groups}

5.1. Preliminaries on virtual endomorphisms of groups. Let $G$ be a transitive state-closed subgroup of $\mathcal{A}(Y)$, where $Y=\{1,2, \ldots, m\}$. Then $\left[G: \operatorname{Fix}_{G}(1)\right]=m$ and the projection on the 1 st coordinate of $\operatorname{Fix}_{G}(1)$ produces a subgroup of $G$; that is, $\pi_{1}: \operatorname{Fix}_{G}(1) \rightarrow G$ is a virtual endomorphism of $G$. This notion has proven to be effective in studying state-closed groups. We give a quick review below.

Let $G$ be a group with a subgroup $H$ of finite index $m$ and a homomorphism $f: H \rightarrow G$. A subgroup $U$ of $G$ is semi-invariant under the action of $f$, provided that $(U \cap H)^{f} \leq U$. If $U \leq H$ and $U^{f} \leq U$ then $U$ is $f$-invariant.

The largest subgroup $K$ of $H$ which is normal in $G$ and $f$-invariant is called the $f$-core $(H)$. If the $f$-core $(H)$ is trivial then $f$ and the triple $(G, H, f)$ are said to be a simple.

Given a triple $(G, H, f)$ and a right transversal $L=\left\{x_{1}, x_{2}, \ldots, x_{m}\right\}$ of $H$ in $G$, the permutational representation $\pi: G \rightarrow \operatorname{Perm}(1,2, \ldots, m)$ is $g^{\pi}: i \rightarrow j$, which 
is induced from the right multiplication $H x_{i} g=H x_{j}$. We produce recursively a representation $\varphi: G \rightarrow \mathcal{A}(m)$ as follows:

$$
g^{\varphi}=\left(\left(x_{i} g \cdot\left(x_{(i) g^{\pi}}\right)^{-1}\right)^{f \varphi}\right)_{1 \leq i \leq m} g^{\pi} .
$$

One further expansion of $g^{\varphi}$ is

$$
\begin{aligned}
g^{\varphi} & =\left(\left(\left(x_{j} g_{i} \cdot x_{(j) g_{i}^{\pi}}^{-1}\right)^{f \varphi}\right)_{1 \leq j \leq m} g_{i}^{\pi}\right)_{1 \leq i \leq m} g^{\pi}, \\
& =\left(\left(\left(x_{j} g_{i} \cdot x_{(j) g_{i}^{\pi}}^{-1}\right)^{f \varphi}\right)_{1 \leq j \leq m}\right)_{1 \leq i \leq m}\left(g_{i}^{\pi}\right)_{1 \leq i \leq m} g^{\pi},
\end{aligned}
$$

where $g_{i}=\left(x_{i} g \cdot x_{(i) g \pi}^{-1}\right)^{f}$.

The kernel of $\varphi$ is precisely the $f$-core $(H), G^{\varphi}$ is state-closed and $H^{\varphi}=$ $\operatorname{Fix}_{G^{\varphi}}(1)$.

5.1.1. Changing transversals. We will show below that changing the transversal of $H$ in $G$ produces another representation of $G$, conjugate to the original one by an explicit automorphism of the $m$-ary tree.

Proposition 3. Let $(G, H, f)$ be a triple and

$$
L=\left\{x_{1}, x_{2}, \ldots, x_{m}\right\}, \quad L^{\prime}=\left\{x_{1}^{\prime}=h_{1} x_{1}, x_{2}^{\prime}=h_{2} x_{2}, \ldots, x_{m}^{\prime}=h_{m} x_{m}\right\}
$$

right transversals of $H$ in $G$ where $h_{i} \in H$. Let $\varphi=\varphi_{x_{i}}, \varphi^{\prime}=\varphi_{h_{i} x_{i}}: G \rightarrow \mathcal{A}(m)$ be the corresponding tree representations and define the following elements of $\mathcal{A}(m)$,

$$
\begin{aligned}
& \gamma=\gamma_{h_{i}, \varphi^{\prime}}=\left(\left(h_{i}\right)^{f \varphi^{\prime}}\right)_{1 \leq i \leq m}, \\
& \lambda=\lambda_{h_{i}, \varphi^{\prime}}=\gamma \gamma^{(1)} \ldots \gamma^{(n)} \ldots
\end{aligned}
$$

Then

$$
\varphi_{h_{i} x_{i}}=\varphi_{x_{i}}\left(\lambda_{h_{i}^{-1}, \varphi_{x_{i}}}\right) .
$$

Proof. The representations $\varphi, \varphi^{\prime}: G \rightarrow \mathcal{A}(m)$ are defined by

$$
\begin{aligned}
g^{\varphi} & =\left(\left(x_{i} g \cdot\left(x_{(i) g \pi}\right)^{-1}\right)^{f \varphi}\right)_{1 \leq i \leq m} g^{\pi}, \\
g^{\varphi^{\prime}} & =\left(\left(x_{i}^{\prime} g \cdot\left(x_{(i) g^{\pi}}^{\prime}\right)^{-1}\right)^{f \varphi^{\prime}}\right)_{1 \leq i \leq m} g^{\pi} .
\end{aligned}
$$

The relationship between $\varphi^{\prime}$ and $\varphi$ is established as follows,

$$
\begin{aligned}
g^{\varphi^{\prime}} & =\left(\left(h_{i} x_{i} g \cdot\left(h_{(i) g^{\pi}} x_{(i) g^{\pi}}\right)^{-1}\right)^{f \varphi^{\prime}}\right)_{1 \leq i \leq m} g^{\pi} \\
& =\left(\left(h_{i}\left(x_{i} g \cdot x_{(i) g^{\pi}}^{-1}\right) h_{(i) g^{\pi}}^{-1}\right)^{f \varphi^{\prime}}\right)_{1 \leq i \leq m} g^{\pi} \\
& =\left(\left(h_{i}\right)^{f \varphi^{\prime}}\right)_{1 \leq i \leq m} \cdot\left(\left(x_{i} g \cdot x_{(i) g^{\pi}}^{-1}\right)^{f \varphi^{\prime}}\right)_{1 \leq i \leq m} \cdot\left(\left(h_{(i) g^{\pi}}^{-1}\right)^{f \varphi^{\prime}}\right)_{1 \leq i \leq m} g^{\pi} \\
& =\left(\left(h_{i}\right)^{f \varphi^{\prime}}\right)_{1 \leq i \leq m} \cdot\left(\left(x_{i} g \cdot x_{(i) g^{\pi}}^{-1}\right)^{f \varphi^{\prime}}\right)_{1 \leq i \leq m} g^{\pi} \cdot\left(\left(h_{i}\right)^{f \varphi^{\prime}}\right)_{1 \leq i \leq m}^{-1} .
\end{aligned}
$$


Therefore

$$
g^{\varphi^{\prime}}=\gamma \cdot\left(\left(x_{i} g \cdot x_{(i) g^{\pi}}^{-1}\right)^{f \varphi^{\prime}}\right)_{1 \leq i \leq m} g^{\pi} \cdot \gamma^{-1},
$$

where $\gamma=\left(\left(h_{i}\right)^{f \varphi^{\prime}}\right)_{1 \leq i \leq m}$ is independent of $g$. Repeating this development for each $g_{i}=\left(x_{i} g \cdot x_{(i) g^{\pi}}^{-1}\right)^{f}$, we find that

$$
g^{\varphi^{\prime}}=\gamma \gamma^{(1)} \cdot\left(\left(\left(x_{j} g_{i} \cdot x_{(j) g_{i}^{\pi}}^{-1}{ }^{f \varphi^{\prime}}\right)_{1 \leq j \leq m} g_{i}^{\pi}\right)_{1 \leq i \leq m} g^{\pi} \cdot \gamma^{-(1)} \gamma^{-1} .\right.
$$

Thus in the limit we obtain $\lambda=\gamma \gamma^{(1)} \ldots \gamma^{(n)} \ldots$ such that

$$
\begin{aligned}
g^{\varphi^{\prime}} & =\lambda g^{\varphi} \lambda^{-1} \quad \text { for all } g \in G, \\
\varphi & =\varphi^{\prime} \lambda .
\end{aligned}
$$

Introducing the explicit dependence of $\varphi, \varphi^{\prime}, \lambda$ on the transversals, the previous equation becomes

$$
\varphi_{x_{i}}=\left(\varphi_{h_{i} x_{i}}\right)\left(\lambda_{h_{i}, \varphi_{h_{i} x_{i}}}\right) .
$$

On replacing $h_{i}$ by $h_{i}^{-1}$ and on denoting $h_{i}^{-1} x_{i}$ by $x_{i}^{\prime}$, we obtain

$$
\varphi_{h_{i} x_{i}^{\prime}}=\left(\varphi_{x_{i}^{\prime}}\right)\left(\lambda_{h_{i}^{-1}, \varphi_{x_{i}^{\prime}}}\right) .
$$

Example 1. Let $G=C=\langle a\rangle$ be the infinite cyclic group, let $H=\left\langle a^{2}\right\rangle$ and let $f: H \rightarrow G$ be defined by $a^{2} \rightarrow a$. Given $l, k \geq 0$, then on choosing the transversal $L_{k, l}=\left\{a^{2 k}, a^{2 l+1}\right\}$ for $H$ in $G$, we obtain the representation $\varphi_{k, l}: G \rightarrow \mathcal{A}(m)$, where $\varphi_{k, l}: a \rightarrow \alpha=\left(\alpha^{k-l}, \alpha^{-k+l+1}\right) \sigma$.

5.1.2. Subtriples, quotient triples. Given a triple $(G, H, f)$ and given subgroups $V \leq G, U \leq H \cap V$ such that $(U)^{f} \leq V$, we call $\left(V, U,\left.f\right|_{U}\right)$ a sub-triple of $G$. If $N$ is a normal semi-invariant subgroup of $G$, then $\bar{f}: \frac{H N}{N} \rightarrow \frac{G}{N}$ given by $\bar{f}: N h \rightarrow N h^{f}$ is well defined and $\left(\frac{G}{N}, \frac{H N}{N}, \bar{f}\right)$ is a quotient triple.

Let $(G, H, f)$ be a simple triple where $G$ is abelian and $[G: H]=m$. Then any sub-triple of $G$ is simple. Let $T=\operatorname{tor}(G)$ denote the torsion subgroup of $G$ and for $l \geq 1$ define $G(l)=\{g \in T|o(g)| l\}, H(l)=G(l) \cap H$. Then, clearly, $f: \operatorname{tor}(H) \rightarrow \operatorname{tor}(G)$ and $f: H(l) \rightarrow G(l)$. Therefore, $\operatorname{tor}(G)$ and $G(l)$ are semi-invariant and $\left(\operatorname{tor}(G), \operatorname{tor}(H),\left.f\right|_{\operatorname{tor}(H)}\right)$ and $\left(G(l), H(l),\left.f\right|_{H(l)}\right)$ are simple sub-triples.

Lemma 2. Let $(G, H, f)$ be a simple triple. The triple $\left(\frac{G}{G(l)}, \frac{H G(l)}{G(l)}, \bar{f}\right)$ is also simple.

Proof. For suppose $K \leq H$ is such that $G(l) K^{f} \leq G(l) K$. Then

$$
\left(G(l) K^{f}\right)^{l}=\left(K^{f}\right)^{l}=\left(K^{l}\right)^{f} \leq(G(l) K)^{l}=(K)^{l} ;
$$

that is, $K^{l}$ is $f$-invariant. Since $f$ is simple, $K^{l}=\{e\}$, and so $K \leq G(l)$. 


\subsection{The torsion subgroup}

Proposition 4. Let $A$ be transitive state-closed abelian group of degree $m$. Then $\operatorname{tor}(A)$ has finite exponent and is therefore a direct summand of $A$.

Proof. Let $T=\operatorname{tor}(A), A_{1}=\operatorname{Stab}_{A}(1), T_{1}=T \cap A_{1}$ and $\left[T: T_{1}\right]=m^{\prime}$. Then the projection on the 1st coordinate of $T_{1}$ is a subgroup of $T$ and the triple $\left(T, T_{1},\left.\pi_{1}\right|_{T_{1}}\right)$ is simple of degree $m^{\prime} \mid m$; let $m=m^{\prime} m^{\prime \prime}$. Hence, in this representation $T$ is a torsion transitive state-closed subgroup of $\mathcal{A}_{m^{\prime}}$, the automorphism group of the tree $\mathcal{T}_{m^{\prime}}$.

Fixing this last representation of $T$, let $Q=P(T)$ and let $\sigma_{i}(1 \leq i \leq k)$ be a minimal set of generators of $Q$ and as before, let $\beta_{i}=\beta\left(\sigma_{i}\right) \in T$ be such that $\sigma\left(\beta_{i}\right)=\sigma_{i}$. Let $r$ be the maximum order of the elements $\beta_{1}, \ldots, \beta_{k}$. As any $\alpha \in T$ can be written in the form

$$
\alpha=\prod_{1 \leq i \leq k} \beta_{i}^{r_{i 1}}\left(\beta_{i}^{r_{i 2}}\right)^{\left(l_{2}\right)} \ldots\left(\beta_{i}^{r_{i j}}\right)^{\left(l_{j}\right)} \ldots,
$$

it follows that $\alpha^{r}=e$.

Since $T$ has finite exponent, it is a pure bounded subgroup of $A$ and therefore it is a direct summand of $A$ ([6], Theorem 4.3.8).

We recall a classic example of an abelian group $G$ which does not split over its torsion subgroup (see [6], p. 108).

Example 2. Let $G$ be the direct product of groups $\prod_{i \geq 1} C_{i}$, where $C_{i}=\left\langle c_{i}\right\rangle$ is cyclic of order $p^{i}$ and let $H$ be the direct sum $\sum_{i \geq 1} C_{i}$. Then $H \leq \operatorname{tor}(G)=\bigcup_{l \geq 1} G\left(p^{l}\right)$. Moreover, $H$ is a basic subgroup of $G$ and in particular, $\frac{G}{H}$ is $p$-divisible. This observation leads directly to a proof that $G$ does not split over tor $(G)$.

The proof of the previous proposition did not establish the exponent of $\operatorname{tor}(A)$. This we do in the next two lemmas.

Lemma 3. Let $m$ be a prime number and $A$ an abelian transitive state-closed torsion group of degree $m$. Then $A$ is conjugate by a tree automorphism to a subgroup of the diagonal-topological closure of $\langle\sigma\rangle$ and so has exponent $m$.

Proof. We observe that $A(m)$ is not contained in $A_{1}=\operatorname{Stab}_{A}(1)$. For otherwise, $A(m)$ would be invariant under the projection on the first coordinate. Choose $a \in$ $A \backslash A_{1}$ of order $m$. Therefore, $A=A_{1}\langle a\rangle$. On choosing $\left\{a^{i} \mid 0 \leq i \leq m-1\right\}$ as a transversal of $A_{1}$ in $A$, the image of $a$ acquires the form $\sigma=(1, \ldots, m)$ in this tree representation of $A$. Thus, we may suppose by Proposition 3 that $\sigma \in A$. Therefore, $\tilde{A}$ contains the subgroup $\langle\widetilde{\sigma}\rangle=\left\langle\sigma^{(i)} \mid i \geq 0\right\rangle$. By Proposition 2, we have $C_{\mathcal{A}}\langle\widetilde{\sigma}\rangle=\langle\sigma\rangle^{*}$ and thus, $A \leq C_{\mathcal{A}}(A) \leq\langle\sigma\rangle^{*}$. 
Lemma 4. Suppose that $A$ is an abelian transitive state-closed torsion group of degree $m$. Then the exponent of $A$ is equal to the exponent of $P(A)$.

Proof. By induction on $|P(A)|=m$. The exponent of $A$ is a multiple of the exponent of $P(A)$. By the previous lemma, we may assume $m$ to be composite. Let $p$ be a prime divisor of $m$ and $A(p)=\left\{a \in A \mid a^{p}=e\right\}$. Then $A(p)$ is a nontrivial subgroup and $P(A(p)) \leq\left\{\sigma \in P \mid \sigma^{p}=e\right\}$. By Lemma $2,\left(\frac{A}{A(p)}, \frac{A_{1} A(p)}{A(p)}, \overline{\pi_{0}}\right)$ is simple; also, $P\left(\frac{A}{A(p)}\right)=\frac{P(A)}{P(A(p))}$. The proof follows by induction.

Theorem 7. Suppose that $A$ is an abelian transitive state-closed torsion group of degree $m$. Then $A$ is conjugate to a subgroup of the topological closure of

$$
\widetilde{P(A)}=\left\langle\sigma^{(i)} \mid \sigma \in P(A), i \geq 0\right\rangle .
$$

Proof. Let $P=P(A)$ have exponent $r$ and let $B$ be a maximal homogeneous subgroup of $P$ of exponent $r$ (that is, $B$ is a direct sum of cyclic groups of order $r$ ), minimally generated by $\left\{\sigma_{i} \mid 1 \leq i \leq s\right\}$. Choose for each $\sigma_{i}$ an element $\beta_{i}=$ $\beta\left(\sigma_{i}\right) \in A$ and let $\dot{B}=\left\langle\beta_{i} \mid 1 \leq i \leq s\right\rangle$. Then, as the order of each $\beta_{i}$ is a multiple of $r$, while the exponent of $A$ is $r$, we conclude from the previous lemma that $o\left(\beta_{i}\right)=o\left(\sigma_{i}\right)=r$ for $1 \leq i \leq s$. Since $\beta_{i} \rightarrow \sigma_{i}$ defines a projection of $\dot{B}$ onto $B$ we conclude that $\dot{B} \cong B$ and $\dot{B} \cap A_{1}=\{e\}$, where $A_{1}=\operatorname{Stab}_{A}(1)$.

Clearly $\dot{B}$ is a pure bounded subgroup and so it has a complement $L$ in $A$, which may be chosen to contain $A_{1}$. Choose a right transversal $W$ of $A_{1}$ in $L$. Then the set $W \dot{B}$ is a right transversal of $A_{1}$ in $A$. With respect to this transversal, the triple $\left(A, A_{1}, \pi_{1}\right)$ produces a transitive state-closed representation $\varphi$ where $\dot{B}^{\varphi}=B$. By Proposition 3, we may rewrite $A^{\varphi}$ as $A$. Then the diagonal-topological closure $A^{*}$ contains $B^{*}$. Let $V$ be a complement of $B$ in $P$. Each $\alpha \in A^{*}$ can be factored as $\alpha=\beta \gamma$, where $\beta \in B^{*}$ and $\gamma$ is such that each of its states $\gamma_{u}$ have activity $\sigma\left(\gamma_{u}\right) \in V$. Therefore, the set of these $\gamma^{\prime}$ 's is a group $\Gamma$ such that $\Gamma=\Gamma^{*}$ and $A^{*}=\Gamma \oplus B^{*}$. Then $\left(\Gamma, \Gamma \cap A_{1}, \pi_{1}\right)$ is a simple triple with $P(\Gamma)$ having exponent smaller than $r$. The proof is finished by induction on the exponent.

The example below illustrates some of the ideas developed so far.

Example 3. Let $m=4, Y=\{1,2,3,4\}$ and let $\sigma$ be the cycle $(1,2,3,4)$. Furthermore, let $\alpha=\left(e, e, e, \alpha^{2}\right) \sigma \in \mathcal{A}(4)$ and let $A=\langle\alpha\rangle$. Then

$$
\begin{aligned}
& \alpha^{2}=\left(\alpha^{2}, e, e, \alpha^{2}\right)(1,3)(2,4), \\
& \alpha^{4}=\left(\alpha^{2}\right)^{(1)}=\alpha^{2 x}, \quad\left(\alpha^{2-x}\right)^{2}=e .
\end{aligned}
$$

Thus $A$ is cyclic, torsion-free, transitive and state-closed; it is, however, not diagonally closed because $\alpha^{x} \notin A$. Even though $A$ is torsion-free, its diagonal closure $\tilde{A}=\left\langle\alpha^{x^{i}} \mid i \geq 0\right\rangle$ is not; for $\kappa=\alpha^{2-x}$ has order 2. Let $K=\left\langle\kappa^{x^{i}} \mid i \geq 0\right\rangle$. 
Then $K \leq \operatorname{tor}(\tilde{A})$ and it is direct to check that $\tilde{A}=\langle\alpha, K\rangle$. Therefore, $K=\operatorname{tor}(\tilde{A})$ and

$$
\tilde{A}=\operatorname{tor}(\tilde{A}) \oplus A \text {. }
$$

Let $Y_{1}=\{1,3\}, Y_{2}=\{2,4\}$. Then $\left\{Y_{1}, Y_{2}\right\}$ is a complete block system for the action of $\alpha$ on $Y$. Also, $\alpha^{2}$ induces the binary adding machine on both $\mathcal{T}\left(Y_{1}\right)$ and $\mathcal{T}\left(Y_{2}\right)$. The topological closure $\bar{A}$ of $A$ is torsion-free and

$$
\operatorname{tor}\left(A^{*}\right)=\operatorname{tor}(\tilde{A}), \quad A^{*}=\operatorname{tor}\left(A^{*}\right) \oplus \bar{A} .
$$

Moreover, tor $\left(A^{*}\right)$ induces a faithful state-closed, diagonally and topologically closed actions on the binary tree $\mathcal{T}\left(Y_{1}\right)$. Therefore, $\operatorname{tor}\left(A^{*}\right)$ is isomorphic to $\frac{\mathbb{Z}}{2 \mathbb{Z}}[[x]]$. Furthermore, $\alpha$ is represented as the binary adding machine on $\mathcal{T}\left(\left\{Y_{1}, Y_{2}\right\}\right)$ and $\bar{A}$ is represented on this tree as the topological closure of the image of $A$.

\section{Cyclic $\mathbb{Z}_{m}[[x]]-$ modules}

Cyclic automorphism groups $\langle\alpha\rangle$ of the tree, for which their state-diagonal-topological closure is isomorphic to a cyclic $\mathbb{Z}_{m}$-module have the form

$$
\alpha=\left(\alpha^{q_{1}}, \ldots, \alpha^{q_{m}}\right) \sigma,
$$

where $q_{i} \in \mathbb{Z}_{m}[[x]]$ for $1 \leq i \leq m$; here

$$
q_{i}=\sum_{j \geq 0} q_{i j} x^{j}, \quad q_{i j}=\sum_{u \geq 0} q_{i j, u} m^{u} \in \mathbb{Z}_{m} .
$$

We prove

Theorem 8. (i) The expression

$$
\alpha=\left(\alpha^{q_{1}}, \ldots, \alpha^{q_{m}}\right) \sigma
$$

is a well-defined automorphism of the m-ary tree.

(ii) Let $A$ be the state closure of $\langle\alpha\rangle$. Then $A^{*}$ is abelian, isomorphic to the quotient ring $\frac{\mathbb{Z}_{m}[[x]]}{(r)}$, where

$$
r=m-q x \text { and } q=q_{1}+\cdots+q_{m} .
$$

Proof. (1) Let $\sigma(l)$ denote the permutation induced by $\alpha$ on the $l$-th level. Then the expression $\alpha=\left(\alpha^{q_{1}}, \ldots, \alpha^{q_{m}}\right) \sigma$ represents

$$
\sigma(1)=\sigma, \quad \sigma(l)=\left(\sigma(l-1)^{\overline{q_{1}}}, \ldots, \sigma(l-1)^{\overline{q_{m}}}\right) \sigma,
$$

where $\overline{q_{i}}=\overline{q_{i 0}}+\overline{q_{i 1}} x+\cdots+\overline{q_{i(l-1)}} x^{l-1}$ and $\overline{q_{i j}}=q_{i j, 0}+q_{i j, 1} m+\cdots+q_{i j, l-1} m^{l-1}$. 
(2.1) The states of $\alpha$ are words in $\alpha^{p}$ for $p \in \mathbb{Z}_{m}[[x]]$. Let $v=\alpha^{l_{1}} \ldots \alpha^{l_{a}}$, $w=\alpha^{n_{1}} \ldots \alpha^{n_{b}} \in A^{*}$. Then clearly $[v, w] \in \operatorname{Stab}_{A}(1)$. We will prove that the entries of $[v, w]$ are products of conjugates of words in elements of the form $\left[\alpha^{s}, \alpha^{t}\right]$ where $s, t \in \mathbb{Z}_{m}[[x]]$.

Clearly $[v, w]$ can be developed into a word in conjugates of $\left[\alpha^{l_{i}}, \alpha^{n_{j}}\right]$.

Write $p=p_{0}+p^{\prime} x, n=n_{0}+n^{\prime} x$. We compute

$$
\begin{aligned}
{\left[\alpha^{p}, \alpha^{n}\right] } & =\left(\left[\alpha^{p_{0}}, \alpha^{n^{\prime} x}\right]\left[\alpha^{p_{0}}, \alpha^{n_{0}}\right]^{\alpha^{n^{\prime} x}}\right)^{\alpha^{p^{\prime} x}}\left[\alpha^{p^{\prime}}, \alpha^{n^{\prime}}\right]^{x}\left[\alpha^{p^{\prime} x}, \alpha^{n_{0}}\right]^{\alpha^{n^{\prime} x}} \\
& =\left[\alpha^{p_{0}}, \alpha^{n^{\prime} x}\right]^{\alpha^{p^{\prime} x}}\left[\alpha^{p^{\prime}}, \alpha^{n^{\prime}}\right]^{x}\left[\alpha^{p^{\prime} x}, \alpha^{n_{0}}\right]^{\alpha^{n^{\prime} x}} .
\end{aligned}
$$

Therefore, we have to check $\left[\alpha^{\xi}, \alpha^{n x}\right]$ where $\xi \in \mathbb{Z}_{m}, n \in \mathbb{Z}_{m}[[x]]$. Write $\xi=$ $\xi_{0}+m \xi^{\prime}$. Then

$$
\left[\alpha^{\xi}, \alpha^{n x}\right]=\left[\alpha^{\xi_{0}+m \xi^{\prime}}, \alpha^{n x}\right]=\left[\alpha^{\xi_{0}}, \alpha^{n x}\right]^{\alpha^{m \xi^{\prime}}}\left[\alpha^{m \xi^{\prime}}, \alpha^{n x}\right] .
$$

Now

$$
\alpha^{\xi_{0}}=\left(v_{\mathbf{1}}, v_{\mathbf{2}}, \ldots, v_{\boldsymbol{m}}\right) \sigma^{\xi_{0}}
$$

where $v_{\boldsymbol{i}}$ are words in $\alpha^{q_{1}}, \ldots, \alpha^{q_{\boldsymbol{m}}}$ and

$$
\alpha^{m}=\left(\alpha^{q_{1}} \ldots \alpha^{q_{\boldsymbol{m}}}, \alpha^{q_{2}} \ldots \alpha^{q_{\boldsymbol{m}}} \alpha^{q_{1}}, \ldots, \alpha^{q_{\boldsymbol{m}}} \alpha^{q_{1}} \ldots \alpha^{q_{\mathbf{m}-1}}\right) .
$$

Therefore,

$$
\left[\alpha^{\xi_{0}}, \alpha^{n x}\right]=\left(\left[v_{\mathbf{1}}, \alpha^{n}\right], \ldots,\left[v_{\boldsymbol{m}}, \alpha^{n}\right]\right)
$$

and similarly

$$
\left[\alpha^{m \xi^{\prime}}, \alpha^{n x}\right]=\left(\left[\left(\alpha^{q_{1}} \ldots \alpha^{q_{\boldsymbol{m}}}\right)^{\xi^{\prime}}, \alpha^{n}\right], \ldots,\left[\left(\alpha^{q_{\boldsymbol{m}}} \alpha^{q_{1}} \ldots \alpha^{q_{\mathbf{m}-1}}\right)^{\xi^{\prime}}, \alpha^{n}\right]\right) .
$$

Now we write $\beta=\alpha^{q_{1}} \ldots \alpha^{q_{m}}$. Then $\left[\beta^{\xi^{\prime}}, \alpha^{n}\right]$ can be developed further, as asserted. The same applies to the other entries.

(2.2) First, clearly $r \alpha=0$. Now let $u=u(x)$ annul $\alpha$; write $u=u_{0}+u^{\prime} x$ where $u_{0}=u(0)$. Then $m \mid u_{0}$ and so

$$
u=m \frac{u_{0}}{m}+u^{\prime} x=(x q) \frac{u_{0}}{m}+u^{\prime} x+v r=x w_{1}+v r
$$

for some $v=v(x)$ and $w_{1}=q \frac{u_{0}}{m}+u^{\prime}$. Then $x w_{1}$ annuls $\alpha$ and so does $w_{1}$. On repeating, we find $w_{i}$ such that $u \equiv x^{i} w_{i} \bmod r$ and $w_{i}$ annuls $\alpha$ for all $i \geq 1$.

In other words, $u \in \bigcap_{n \geq 1}(x \mathbb{Z})^{n}+(r)=(r)$.

The group $D_{\boldsymbol{m}}(\boldsymbol{j})$. Recall $\alpha=\left(e, \ldots, e, \alpha^{x^{j-1}}\right) \sigma \in \mathcal{A}_{m}$. Then $\alpha^{m}=\alpha^{x^{j}}$; that is, $\alpha^{r}=e$ where $r=m-x^{j}$. The states of $\alpha$ are $\alpha, \alpha^{x}, \ldots, \alpha^{x^{j-1}}$ and

$$
D_{m}(j)=\left\langle\alpha, \alpha^{x}, \ldots, \alpha^{x^{j-1}}\right\rangle ;
$$

therefore $D_{m}(j)$ is diagonally closed. The topological closure $\overline{D_{m}(j)}$ is isomorphic to the quotient ring $S=\frac{\mathbb{Z}_{m}[[x]]}{(r)}$, which is clearly a free $\mathbb{Z}_{m}$-module of rank $j$. 


\subsection{The case $P(A)$ cyclic of prime order}

Theorem 9. Let $m$ be a prime number. Let $A$ be a torsion-free abelian transitive stateclosed subgroup of $\mathcal{A}_{m}$. Let $\beta \in A \backslash \operatorname{Stab}_{A}(j)$. Then $A^{*}=\langle\beta\rangle^{*}$ and is topologically finitely generated. Furthermore, $A^{*}$ is conjugate to $\overline{D_{m}(j)}$ for some $j \geq 1$.

The proof is developed in four steps.

Step 1 . For $z \in A$, define $\zeta(z)=j$ such that $z^{m} \in \operatorname{Stab}(j) \backslash \operatorname{Stab}(j+1)$. As $A$ is torsion-free, $\zeta(z)$ is finite for all nontrivial $z$ and $z^{m}=(v)^{(j)}, v \in A \backslash \operatorname{Stab}_{A}(1)$.

Choose $\beta=\left(\beta_{1}, \beta_{2}, \ldots, \beta_{m}\right) \sigma \in A \backslash \operatorname{Stab}_{A}(1)$ having minimum $\zeta(\beta)=j$. If $z \in \operatorname{Stab}_{A}(1), z \neq e$, then there exists $l>0$ such that $z^{m}=(c)^{(l)}$ and $c \in$ $A \backslash \operatorname{Stab}_{A}(1)$. Therefore, by minimality of $\beta$ we have $\zeta(c) \geq \zeta(\beta)$ and $\zeta(z)>\zeta(\beta)$.

Lemma 5 (Uniform gap). Let $z \in \operatorname{Stab}_{A}(1)$. Then $\zeta(z \beta)=\zeta(\beta)$.

Proof. First note that

$$
\begin{aligned}
\beta^{m} & =\left(\beta_{1} \beta_{2} \ldots \beta_{m}\right)^{(1)}, \\
\beta_{1} \beta_{2} \ldots \beta_{m} & =(\gamma)^{(j-1)}, \quad \gamma \in A \backslash \operatorname{Stab}_{A}(1) .
\end{aligned}
$$

We have $z=c^{(1)}$ and $z \beta=\left(c \beta_{1}, c \beta_{2}, \ldots, c \beta_{m}\right) \sigma,(z \beta)^{m}=(u)^{(1)}$, where $u=$ $c^{m} \beta_{1} \ldots \beta_{m}=c^{m}(\gamma)^{(j-1)}$. If $c \in A \backslash \operatorname{Stab}_{A}(1)$ then $\zeta(c)=n \geq j, c^{m} \in$ $\operatorname{Stab}(n) \backslash \operatorname{Stab}(n+1)$, and so, $u \in \operatorname{Stab}_{A}(j-1) \backslash \operatorname{Stab}_{A}(j)$. If $c \in \operatorname{Stab}_{A}(1)$ then $\zeta(c)>j$ and so $c^{m} \in \operatorname{Stab}(k)$, where $k>j$ and again $u \in \operatorname{Stab}(j-1) \backslash \operatorname{Stab}(j)$.

Step 2. Note that

$$
\begin{aligned}
\beta^{m} & =(\gamma)^{(j)}, \quad \gamma^{m}=(\lambda)^{(j)}, \\
\beta^{m^{2}} & =(\lambda)^{(2 j)},
\end{aligned}
$$

where, by the uniform gap lemma above, $\gamma, \lambda \in A \backslash \operatorname{Stab}_{A}(1)$. Therefore, repeating this process, we find that $\beta^{m^{s}}$ induces $\sigma^{(s j)}$ on the $(s j)$-th level of the tree for all $s \geq 0$. Now given a level $t \geq 0$, dividing $t$ by $j$, we get $t=s j+i$ with $0 \leq i \leq j-1$, and then $\left(\beta^{(i)}\right)^{m^{s}}=\left(\beta^{m^{s}}\right)^{(i)}$ induces $\left(\sigma^{(s j)}\right)^{(i)}=\sigma^{(s j+i)}=\sigma^{(t)}$ on the $t$-th level of the tree. It follows that the group $A$ is a subgroup of the topological closure of $\left\langle\beta, \beta^{(1)}, \ldots, \beta^{(j-1)}\right\rangle$.

Step 3. We have for $\beta=\left(\beta_{1}, \beta_{2}, \ldots, \beta_{m}\right) \sigma$,

$$
\beta_{i}=\beta^{p_{i}}, \quad p_{i}=r_{i 0}+r_{i 1} x+\cdots+r_{i(j-1)} x^{j-1} \in \mathbb{Z}_{m}[x],
$$

and

$$
\begin{aligned}
\beta^{m} & =\left(\beta_{1} \beta_{2} \ldots \beta_{m}\right)^{(1)}, \\
\beta_{1} \beta_{2} \ldots \beta_{m} & =\beta^{p_{1}+\cdots+p_{m}}, \\
p_{1}+\cdots+p_{m} & =q \cdot x^{j-1},
\end{aligned}
$$


where $q$ is an invertible element of $\mathbb{Z}_{m}[[x]]$.

Proposition 5. The element $\beta=\left(\beta_{1}, \beta_{2}, \ldots, \beta_{m}\right) \sigma$ is conjugate in $\mathcal{A}_{m}$ to $\alpha=$ $\left(e, \ldots, e, \alpha^{x^{j-1}}\right) \sigma$.

Proof. Let $h=\left(h_{1}, h_{2}, \ldots, h_{m}\right)$ be an automorphism of the tree. Then

$$
\beta^{h}=\left(h_{1}^{-1} \beta_{1} h_{2}, h_{2}^{-1} \beta_{2} h_{3}, \ldots, h_{m}^{-1} \beta_{m} h_{1}\right) \sigma .
$$

Therefore $\beta^{h}=\alpha$ holds if and only if

$$
h_{2}=\beta_{1}^{-1} h_{1}, \quad h_{3}=\beta_{2}^{-1} h_{2}, \ldots, h_{m}=\beta_{m-1}^{-1} h_{m-1}, \quad h_{1}=\beta_{m}^{-1} h_{m} \alpha^{x^{j-1}} .
$$

These conditions can be rewritten as

$$
\begin{gathered}
h_{2}=\beta_{1}^{-1} h_{1}, \quad h_{3}=\beta_{2}^{-1} \beta_{1}^{-1} h_{1}, \ldots, h_{m}=\beta_{m-1}^{-1} \ldots \beta_{1}^{-1} h_{1}, \\
h_{1}=\beta_{m}^{-1} \beta_{m-1}^{-1} \ldots \beta_{1}^{-1} h_{1} \alpha^{x^{j-1}},
\end{gathered}
$$

or as

$$
\begin{aligned}
h & =\left(h_{1}, \beta_{1}^{-1} h_{1}, \beta_{2}^{-1} \beta_{1}^{-1} h_{1}, \ldots, \beta_{m-1}^{-1} \ldots \beta_{1}^{-1} h_{1}\right) \\
& =\left(e, \beta_{1}^{-1}, \beta_{2}^{-1} \beta_{1}^{-1}, \ldots, \beta_{m-1}^{-1} \ldots \beta_{1}^{-1}\right)\left(h_{1}\right)^{(1)},
\end{aligned}
$$

and

$$
\left(\beta_{1} \beta_{2} \ldots \beta_{m}\right)^{h_{1}}=\alpha^{x^{j-1}} .
$$

Since

$$
\beta_{1} \beta_{2} \ldots \beta_{m}=\beta^{q \cdot x^{j-1}},
$$

we repeat the above procedure replacing $\beta$ by $\beta^{q}$ and replacing $h_{1}$ by $\left(h_{1}^{\prime}\right)^{x^{j-1}}$. This leads to the conjugation equation

$$
\left(\beta^{q}\right)^{h_{1}^{\prime}}=\alpha .
$$

In this manner, we determine an automorphism $h$ of the tree which effects the required conjugation

$$
\beta^{h}=\alpha .
$$

Example 4. Let $\beta=\left(e, \beta^{q}\right) \sigma$, where $q=1+x$. Then $\beta$ is conjugate to the adding machine $\alpha=(e, \alpha) \sigma$. Note that from Example 1, $\beta$ is not obtainable from $\alpha$ by simply choosing a different transversal. To exhibit the conjugator $h: \beta \rightarrow \alpha$ constructed in the proof, define the polynomial sequences

$$
\begin{aligned}
c_{0}=1, & c_{1}=q, \quad c_{n}=2 c_{n-2}+c_{n-1} ; \\
c_{-1}^{\prime}=0, & c_{0}^{\prime}=0, \quad c_{n}^{\prime}=c_{n-1}+c_{n-1}^{\prime} .
\end{aligned}
$$

Then

$$
h=(e, e)^{(0)}\left(e, \beta^{-1}\right)^{(1)}\left(e, \beta^{-(1+q)}\right)^{(2)} \ldots\left(e, \beta^{-c_{n}^{\prime}}\right)^{(n)} \ldots
$$


Step 4. By Proposition 2, we have $A \leq \bar{A}=C_{\mathcal{A}}(\alpha)$ and

$$
A^{h} \leq C_{\mathcal{A}}\left(\alpha^{h}\right)=C_{\mathcal{A}}(\beta)=\overline{D_{m}(j)} .
$$

This finishes the proof of the theorem.

\section{References}

[1] A. Berlatto and S. Sidki, Virtual endomorphisms of nilpotent groups. Groups Geom. Dyn. 1 (2007), 21-46. Zbl 1128.20016 MR 2294246

[2] A. M. Brunner and S. Sidki, On the automorphism group of the one-rooted binary tree. $J$. Algebra 195 (1997), 465-486. Zbl 0902.20017 MR 1469633

[3] V. Nekrashevych, Self-similar groups. Math. Surveys Monogr. 117, Amer. Math. Soc., Providence, RI, 2005. Zbl 1087.20032 MR 2162164

[4] V. Nekrashevych and S. Sidki, Automorphisms of the binary tree: state-closed subgroups and dynamics of 1/2-endomorphisms. In Groups: topological, combinatorial and arithmetic aspects, London Math. Soc. Lecture Note Ser. 311, Cambridge University Press, Cambridge 2004, 375-404. Zbl 1144.20305 MR 2073355

[5] L. Ribes and P. Zalesskii, Profinite groups. Ergeb. Math. Grenzgeb. (3) 40, Springer-Verlag, Berlin 2000. Zbl 0949.20017 MR 1775104

[6] D. J. S. Robinson, A course in the theory of groups. 2nd ed., Grad. Texts in Math. 80, Springer-Verlag, New York 1996. Zbl 0836.20001 MR 1357169

Received July 20, 2009; revised October 2, 2009

A. M. Brunner, Department of Mathematics, University of Wisconsin-Parkside, Kenosha, Wisconsin 53141-2000, U.S.A.

E-mail: brunner@uwp.edu

S. N. Sidki, Departamento de Matematica, Universidade de Brasília, Brasília DF 70910900, Brazil

E-mail: sidki@mat.unb.br 